\title{
Energy Efficiency Optimization in D2D-Cellular Converged Networks for Video Delivery
}

\author{
Meiping Jia, ${ }^{1}$ Chao Zhang, ${ }^{1,2}$ and Yun Rui ${ }^{2}$ \\ ${ }^{1}$ School of Electronics and Information Engineering, Xian Jiaotong University, Xi'an 710049, China \\ ${ }^{2}$ National Mobile Communications Research Laboratory, Southeast University, Nanjing, China \\ Correspondence should be addressed to Chao Zhang; chaozhang@mail.xjtu.edu.cn
}

Received 30 April 2016; Revised 17 August 2016; Accepted 18 September 2016

Academic Editor: Bo Rong

Copyright (c) 2016 Meiping Jia et al. This is an open access article distributed under the Creative Commons Attribution License, which permits unrestricted use, distribution, and reproduction in any medium, provided the original work is properly cited.

\begin{abstract}
A new paradigm of wireless video delivery between users in the converged D2D network and cellular system is studied in this paper. D2D communication enables direct data transmissions of nearby user equipment (UE) by sharing the same time and frequency resource, thereby improving spectrum utilization, system throughput, and energy efficiency. Yet in the case that D2D network cannot provide the requested video files for the limited storage capacity of UE, users have to access the video files in the traditional cellular mode. This paper investigates a D2D-cellular converged network where users are scattered over the area of a single cell according to the spatial Poisson Point Processes and D2D users are able to store part of video files requested by other users according to file popularity. First, we derive the energy efficiency of the D2D-cellular converged network. Then, to maximize the energy efficiency of the D2D-cellular converged network, the optimal collaborative distance of D2D communication and the number of cache files are addressed. Finally, via simulations, we show the effects of these system parameters on energy efficiency and point out the existence of optimal solutions.
\end{abstract}

\section{Introduction}

With the rapid development of wireless communication technology, the users pursuing video delivery are growing greatly. At present, the growing popularity of smartphones and tablets with built-in support for multimedia contents has opened the possibility for watching long video at high resolution on mobile device. Recent studies have showed that the traffic generated by video request delivery will soon exceed other mobile internet business [1]. In order to improve the satisfaction of consumers, we have to open a new business mode to solve the burden of video delivery problem in the cellular network.

It is found that most users in the cell focus on only a small percentage of popular video files [2]. What is more, the traffic generated by the neighboring users is becoming larger and larger. Recently, Device-to-Device (D2D) communication is deemed to be fit for the developing trend and related features, whereas this technology also has some limitations, such as long-distance transmission. The converged network of D2D communication and the traditional cellular system is an effective way to solve the contradiction between the rapid growth of video business data traffic and limited capacity of wireless network transmission [2]. For example, the D2D assisted cellular systems are applied for the public safety applications to transmit surveillance video $[3,4]$. The research of D2D-cellular converged network is still in its infancy. The D2D communication energy efficiency problems of converged network are studied in [5-8]. The converged network where the traditional cellular spectrum is shared with multiple D2D communication links is proposed in [9]. However, these literatures do not consider D2D requirements under the practical application scenarios and specific business requirements. Simply raising throughput and energy efficiency of settled and existing D2D link will not surely bring performance improvement under specific business in the converged network.

How to schedule the D2D transmission links with specific application scenarios and business requirements in the converged system is still a challenge. Nowadays, the most of mobile devices have been equipped with memory modules of large storage capacity. Thus, users are able to store popular 


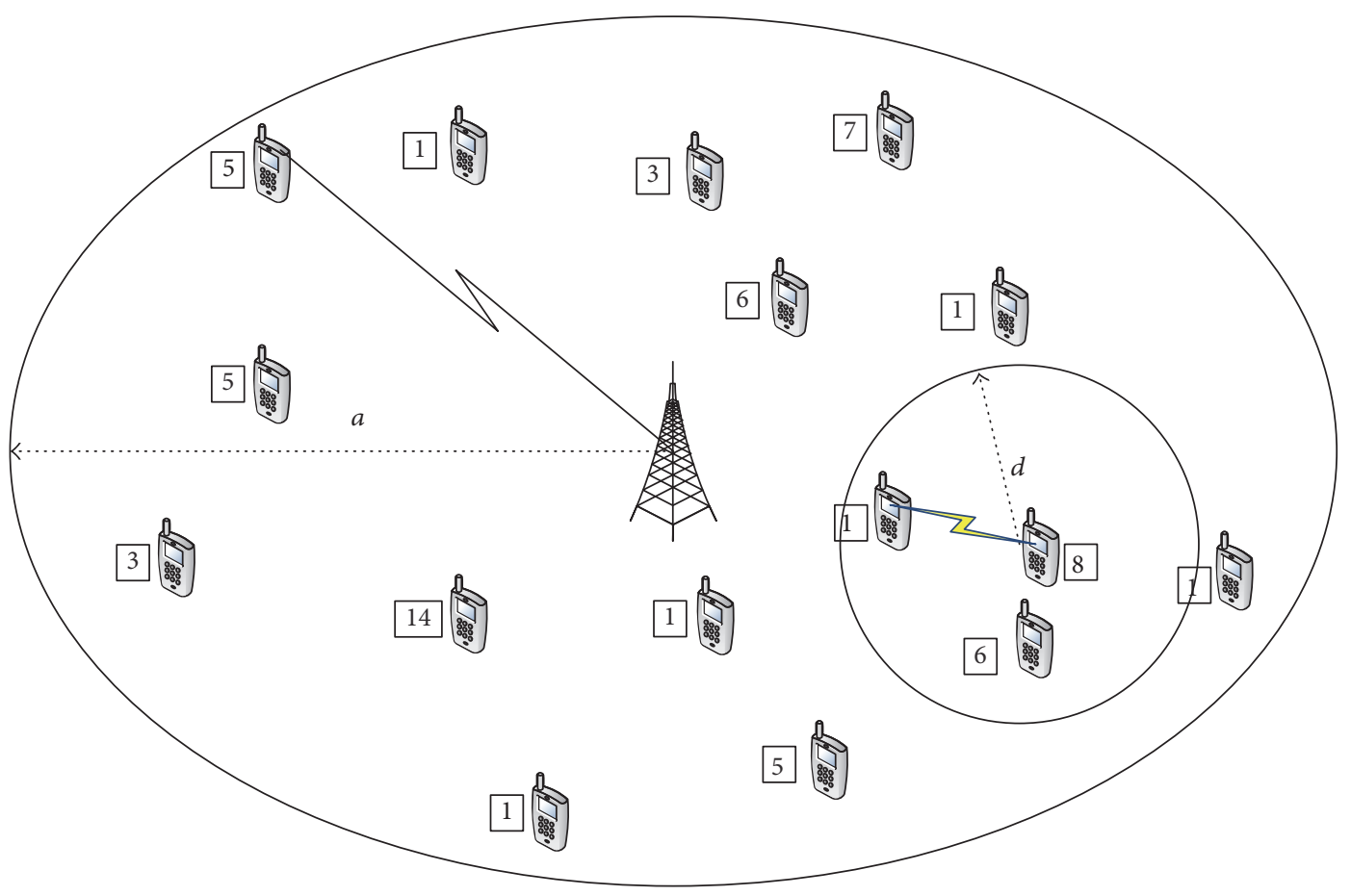

Figure 1: System model.

video files in their devices and share those files with other users. In [10], mobile switching center is introduced into the cellular system to store the popular files. However, it only helps to reduce the strain on the cellular backhaul, without avoiding the "on air" congestion. In the view of sharing multimedia data, authors of [11] propose a user head node based converged scheme, in which the user head node stores multimedia data and distributes it to each user of the multicast group by the D2D links. The users' requirements of video files are not taken into account in the abovementioned literatures. Considering the users' request, Golrezaei et al. propose a new paradigm for wireless video sharing, where users can store popular video files and share files via D2D transmission [2]. However, the important feature that unbalanced characteristic of users' request is not exploit to improve the system efficiency.

The main contributions of this paper are summarized as follows: On the one hand, considering specific application scenarios and business requirements, we establish a new paradigm of wireless video delivery between users in the D2D-cellular converged network, where users store video files according to popularity and follow a spatial Poisson Point Process (PPP). Upon request, the cache files are transmitted to the user via either D2D link or traditional cellular link. If the requested file is stored in the nearby users within the $\mathrm{D} 2 \mathrm{D}$ communication range, D2D communication is used to deliver that file. Otherwise, the user has to access the BS to obtain the file. On the other hand, we derive the energy efficiency of the converged network and set up two optimization problems to seek the optimal collaborative distance and the number of cache files in the large virtual cache.

\section{System Model}

2.1. Converged Network Model. The framework of the proposed D2D-cellular converged network is depicted in Figure 1. The converged network consists of two parts: a D2D communication network and a cellular network. For simplicity, we assume that the cell is round and only consider one cell in this paper. The multicell scenario is planned to be studied in future work. Hence, there is no intercell interference in our system. We further assume that the D2D communication does not interfere with these links between BS and users. To meet the high-rate video file transmission, we herein assume the D2D communication works in the overlay mode, where the D2D links incur no interference to the cellular links. Users in the cell are randomly distributed over a circular range with radius $a$ and users move according to the distribution of a spatial Poisson Point Processes (PPP) with average intensity $\rho$ arrivals $/ \mathrm{m}^{2}$ [12]. Users in the cell can request popular contents from neighboring users through D2D communication, whereas they can also request contents from the BS by the traditional cellular communication. Users may request files from a set of $m$ files, named "library." We assume that each user stores exactly one file. This assumption yields a clean formulation and can be easily extended for larger storage capacities. Users' devices pool their caching resources to create a larger virtual cache to store video files. The BS is aware of the stored files and channel state information of the users and controls the D2D communications [2]. D2D communications adopt the centralized scheduling scheme and BS can allocate subchannels for D2D user pairs to avoid interference. For example, user node $u$ establishes a link with BS when user node $u$ requests a file from the 
"library" of size $m$. Then, BS searches the requested file in the certain area where the user is located at the center point. If the file is found, BS allocates subchannels for the D2D link between the user $u$ and the user storing the file. Otherwise, the BS establishes a link between itself and the user $u$. In most of D2D communication literatures, the transmission range of $\mathrm{D} 2 \mathrm{D}$ link is limited by the so-called collaboration distance. D2D link can be set up between two users with a distance smaller than the collaboration distance. We define the maximum allowable distance $d$ as the D2D collaboration distance, which is determined by the transmission power of D2D communication.

2.2. Files Caching Model. The converged network provides video files to the users who have a demand of these files, which can be seemed as a service system. We assume that there are $m$ files in the service system. Considering the redundancy of user requests, each user stores a file according to popularity of files independently. In other words, the more popular the file is, the larger the number cached by users is. Zipf distribution has been established as a good model to measure the popularity of files [13]. In this paper, we also use Zipf distributions to model the distribution of video file popularity and user requests. Then, the probability that users cache the $i$ th ranked file can be expressed as

$$
p_{i}=\frac{(1 / i)^{r_{c}}}{\sum_{j=1}^{m}(1 / j)^{r_{c}}}, \quad \text { for } i=1,2, \ldots, m
$$

where $r_{c} \geq 0$ is a parameter named skew coefficient and characterizes the distribution by controlling the relative popularity. For $r_{c}=0$, the Zipf distribution becomes the uniform distribution. For $r_{c}=1$, the Zipf distribution is in line with the 80-20 principle; that is, 80 percent of requests are for 10-20 percent of video files. In Figure 2, we show the Zipf distributions with $r_{c}=0.2,0.4,0.8$. As a result, most of users store few top-ranking files which may be delivered by D2D links with a very high probability. Therefore, it effectively improves the transmission bandwidth utilization. Through the abovementioned assumptions, user nodes need to satisfy the following two conditions to form D2D user pairs: (1) one activated user exists within a distance of $d$ from the requesting user. (2) The activated user caches the required file.

\section{Energy Efficiency Analysis}

In order to clearly describe the progress of converged network delivering files to users, we assume a reference user A requests a specific file as shown in Figure 1. User A firstly sends the request to the $B S$ and then $B S$ searches the file within a distance of $d$. If the file is stored by the users in this circle, assume the nearest user B has this file; BS will allocate channel resource block to users A and B to form a D2D link as [14]. Otherwise, user A receives the file through the traditional cellular network.

We cannot accurately get the location of users due to mobility of users. So, we use the Poisson Point Processes (PPP) to draw the distribution of mobile users. We assume

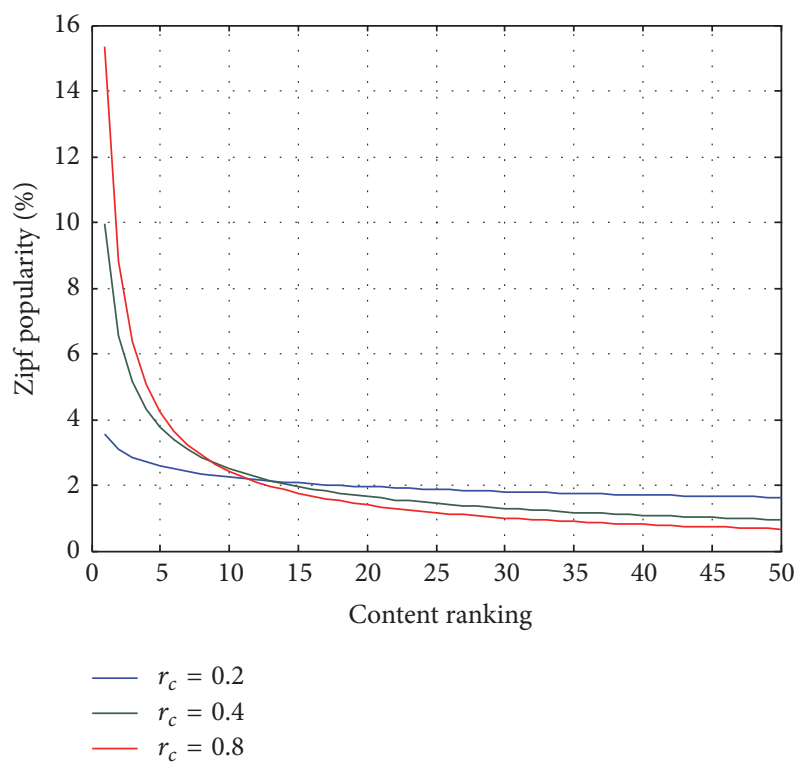

FIGURE 2: Zipf distributions with different exponents.

mobile users follow PPP with average intensity $\rho$; that is, the probability that $l$ users exist in the area $S$ is

$$
f(l ; S)=\frac{(|S| \rho)^{l}}{l !} e^{-|S| \rho}
$$

where $|S|$ means the area of district $S$ [15]. Denote the probability that the user stores the file $i$ as $p_{i}$. Therefore, the file $i$ is distributed with the PPP model with average intensity $\rho p_{i}$. Let $D$ be a random variable representing the distance between the reference user requesting file $i$ and the nearest user storing the file $i$. Thus, the coverage area $S$ of D2D communication is a circular with radius $D$. In other words, when $D>d$, there exists no user with file $i$ in the area $S$; that is, $|S|=\pi d^{2}$. According to (2), the probability of the reference user successfully establishing a D2D link to deliver the desired file is

$$
\begin{aligned}
\operatorname{Pr}(D<d) & =1-\operatorname{Pr}(D>d)=1-f(0 ; d) \\
& =1-e^{-|S| \rho p_{i}} .
\end{aligned}
$$

Thus, the D2D link between two users is activated [16]. Obviously, only users without file $i$ try to send the request to get file $i$. As a result, averagely, there are $\rho\left|S^{\prime}\right|\left(1-p_{i}\right)$ users who may request file $i$ in the cell. Herein, $\left|S^{\prime}\right|$ is the coverage area $S^{\prime}$ of the cellular system.

Without loss of generality, we assume that the file with a smaller index has a larger probability of being requested by a user. Let $q_{i}$ denote the probability that file $i$ is requested by a user; that is, $q_{i} \geq q_{j}$ if $i<j$. As the popularity of files comes from the statistical results of user requests, the user request probability also follows the Zipf distribution. Specifically, we model the user requests as the Zipf distribution with exponent $r_{q}$, which is not necessarily equal to $r_{c}$. 
3.1. Number of D2D Links. The probability of file $i$ requested by a user can be derived on the basis of (1). Further, the number of users requesting file $i$ can be formulated as

$$
n_{i}=\rho\left|S^{\prime}\right|\left(1-p_{i}\right) q_{i} .
$$

And the number of activated D2D links for delivering file $i$ is

$$
n_{i}=\rho\left|S^{\prime}\right|\left(1-p_{i}\right) q_{i}\left(1-e^{-|S| \rho p_{i}}\right) .
$$

From (5), the expected number of active D2D links for all files can be given by

$$
N_{\mathrm{D} 2 \mathrm{D}}=\sum_{i=1}^{m} \rho\left|S^{\prime}\right|\left(1-p_{i}\right) q_{i}\left(1-e^{-|S| \rho p_{i}}\right) .
$$

3.2. Number of Cellular Links. The reference user sends a request to $\mathrm{BS}$ for requesting file $i$. Then, BS chooses the traditional manner to set up a cellular link with the reference user if establishing D2D communication link is failure. It implies that traditional cellular link has to be established if the reference receiver cannot find the corresponding user who stores the desired file within the maximum transmission range of D2D links. By (3), the probability of a traditional cellular link that should be established for transmitting file $i$ is

$$
\operatorname{Pr}(D>d)=e^{-|S| \rho p_{i}} .
$$

In this case, the expected number of traditional cellular links for file $i$ is given by

$$
n_{\mathrm{BS}}=\rho\left|S^{\prime}\right|\left(1-p_{i}\right) q_{i} e^{-|S| \rho p_{i}}
$$

Thus, the expected number of users who obtain their desired files in the traditional manner is

$$
N_{\mathrm{BS}}=\sum_{i=1}^{m} \rho\left|S^{\prime}\right|\left(1-p_{i}\right) q_{i} e^{-|S| \rho p_{i}} .
$$

3.3. Energy Efficiency of Converged Network. Regardless of the reference user selecting either D2D mode or cellular mode, the locations of reference receiver and the closest user with desired file are difficult to be determined. In this paper, we use the maximum communication distance to stand for the distance of two pieces of UE. Thus, the collaboration distance $d$ is the communication distance for D2D mode. Let the radius of the cellular network be equal to $a$; that is, $\left|S^{\prime}\right|=\pi a^{2}$, which also is deemed as the communication distance for cellular mode.

Let $\alpha$ be the path loss factor of the wireless signal transmission. The average spectral efficiency in the D2D mode can be given by

$$
C_{\mathrm{D} 2 \mathrm{D}}=\mathbb{E}\left\{\log \left(1+\frac{P_{d}|h|^{2} d^{-\alpha}}{\sigma_{n}^{2}}\right)\right\} .
$$

And the average spectral efficiency in the cellular mode is

$$
C_{\mathrm{BS}}=\mathbb{E}\left\{\log \left(1+\frac{P_{t}|h|^{2} a^{-\alpha}}{\sigma_{n}^{2}}\right)\right\},
$$

where $\mathbb{E}\{\cdot\}$ denotes expectation operator, $P_{d}$ and $P_{t}$ denote the transmit powers of D2D link and BS, $h$ denotes channel gain and follows complex Gaussian distribution $\mathscr{C} \mathcal{N}(0,1)$, and $\sigma_{n}^{2}$ denotes the power of the additive Gaussian noise. It is easy to know that $|h|^{2}$ follows exponential distributed with $\lambda=1 / 2$; that is, the probability density function of $\left|h^{2}\right|$ is $(1 / 2) e^{-x / 2}$. Then, the average spectral efficiency in the D2D mode can be written by

$$
\begin{aligned}
C_{\mathrm{D} 2 \mathrm{D}} & =\int_{0}^{+\infty} \frac{1}{2} \log \left(1+\frac{P_{d} d^{-\alpha} x}{\sigma_{n}^{2}}\right) e^{-x / 2} d x \\
& =-\frac{1}{\ln 2} e^{\sigma_{n}^{2} d^{\alpha} / 2 P_{d}} E_{i}\left(-\frac{\sigma_{n}^{2} d^{\alpha}}{2 P_{d}}\right),
\end{aligned}
$$

where $E_{i}(x)=\int_{-\infty}^{x}\left(e^{t} / t\right) d t$. In the same way, the average spectral efficiency in the cellular mode is

$$
C_{\mathrm{BS}}=-\frac{1}{\ln 2} e^{\sigma_{n}^{2} a^{\alpha} / 2 P_{t}} E_{i}\left(-\frac{\sigma_{n}^{2} a^{\alpha}}{2 P_{t}}\right) .
$$

Energy efficiency (EE), an important criterion for designing green wireless system, is defined as the number of bits that can be transmitted with per energy consumption. Then, we can obtain average EE for a user in the D2D mode and in the cellular mode, respectively:

$$
\begin{aligned}
\eta_{\mathrm{D} 2 \mathrm{D}} & =\frac{-(1 / \ln 2) e^{\sigma_{n}^{2} d^{\alpha} / 2 P_{d}} E_{i}\left(-\sigma_{n}^{2} d^{\alpha} / 2 P_{d}\right)}{P_{d}+P_{u}}, \\
\eta_{\mathrm{BS}} & =\frac{-(1 / \ln 2) e^{\sigma_{n}^{2} a^{\alpha} / 2 P_{t}} E_{i}\left(-\sigma_{n}^{2} a^{\alpha} / 2 P_{t}\right)}{P_{t}+P_{c}},
\end{aligned}
$$

where $P_{u}$ and $P_{c}$ are constant circuit powers consumed by the relevant electronic devices for user and BS, respectively. Accordingly, the EE of the D2D-cellular converged network can be expressed as

$$
\eta=N_{\mathrm{D} 2 \mathrm{D}} \times \eta_{\mathrm{D} 2 \mathrm{D}}+N_{\mathrm{BS}} \times \eta_{\mathrm{BS}}
$$

\section{Optimization Problem}

In this section, we try to maximize the EE of the converged network. Obviously, optimizing $\eta$ in (15) is a joint optimization problem on various system parameters. But it is difficult to solve this problem directly. Herein, we study two key parameters, the collaborative distance $d$ and the number of cache files $n$, to maximize the energy efficiency of the converged network.

4.1. The Collaboration Distance. It is known that the maximum allowable distance for D2D communication is determined by the power level for each transmission. We assume that any transmission pairs with range $d$ can communicate with each other by the same power. In addition, it is not sufficient that the distance between two users of a D2D link should be less than the collaboration distance $d$. We assume 
that users store $m$ files in the virtual cache. Substitute (6), (9), and (14) into (15); we can get the following:

$$
\begin{aligned}
\eta(r) & =\sum_{i=1}^{m} \pi a^{2} \rho\left(1-p_{i}\right) q_{i}\left(-\frac{1}{\ln 2}\right) \\
& \left\{\frac{\left(1-e^{-\pi r^{2} a^{2} \rho p_{i}}\right) e^{\sigma_{n}^{2} r^{\alpha} a^{\alpha} / 2 P_{d}} E_{i}\left(-\sigma_{n}^{2} r^{\alpha} a^{\alpha} / 2 P_{d}\right)}{\left(P_{d}+P_{u}\right)}\right. \\
+ & \left.\frac{e^{-\pi r^{2} a^{2} \rho p_{i}} e^{\sigma_{n}^{2} a^{\alpha} / 2 P_{t}} E_{i}\left(-\sigma_{n}^{2} a^{\alpha} / 2 P_{t}\right)}{\left(P_{t}+P_{c}\right)}\right\} .
\end{aligned}
$$

Clearly, $\eta$ captures a fundamental tradeoff between D2D collaboration distance and the number of activated D2D links. For our theoretical derivations, we define $r=d / a$, $0<r<1$. Thus, finding the optimal $r$ is equal to seeking the optimal $d$. So, we have the conditional optimization problem:

$$
\begin{aligned}
\arg \max _{r} & \eta(r) \\
\text { s.t. } & 0<r<1 .
\end{aligned}
$$

Then, we take a derivative of $\eta$ with respect to $r$, which is expressed in the following:

$$
\begin{aligned}
& \frac{\partial \eta(r)}{\partial r}=\sum_{i=1}^{m} \pi a^{2} \rho\left(1-p_{i}\right) q_{i}\left(-\frac{1}{\ln 2}\right) \\
& \cdot\left\{2 r X e ^ { - r ^ { 2 } X } \left[\frac{e^{r^{\alpha} Y / P_{d}} E_{i}\left(-r^{\alpha} Y / P_{d}\right)}{\left(P_{d}+P_{u}\right)}\right.\right. \\
& \left.-\frac{e^{Y / P_{t}} E_{i}\left(-Y / P_{t}\right)}{\left(P_{t}+P_{c}\right)}\right] \\
& +\frac{\left(1-e^{-X r^{2}}\right)\left(\alpha r^{\alpha-1} Y / P_{d}\right) e^{Y r^{\alpha} / P_{d}} E_{i}\left(-Y r^{\alpha} / P_{d}\right)}{\left(P_{d}+P_{u}\right)} \\
& \left.-\frac{\alpha\left(1-e^{-X r^{2}}\right)}{r\left(P_{d}+P_{u}\right)}\right\},
\end{aligned}
$$

where $X=\pi a^{2} \rho p_{i}$ and $Y=\sigma_{n}^{2} a^{\alpha} / 2$. To solve this problem, we should get the solution of the equation $\partial \eta(r) / \partial r=0$. From (18), finding $r_{\text {opt }}$ analytically in a closed form seems infeasible. Therefore, we can apply Newton's method to seek the optimal D2D collaboration distance.

4.2. Number of Cache Files. In this subsection, we assume that $n$ top-ranking files are stored in the D2D communication network. Users request one of the $n$ files via either the D2D communication link or the traditional cellular link, while the remaining $m-n$ files are transmitted through the cellular system.
The average number of active D2D links for $n$ files can be expressed as

$$
N_{\mathrm{D} 2 \mathrm{D}}=\sum_{i=1}^{n} \rho\left|S^{\prime}\right|\left(1-p_{i}\right) q_{i}\left(1-e^{-|S| \rho p_{i}}\right) .
$$

Similarly, the number of users who obtain their desired file from the BS for $n$ files can be expressed as

$$
N_{\mathrm{BS} 1}=\sum_{i=1}^{n} \rho\left|S^{\prime}\right|\left(1-p_{i}\right) q_{i} e^{-|S| \rho p_{i}} .
$$

Since remaining $m-n$ files are not stored in the virtual cache, they can be delivered to the users by the cellular links. Therefore, the number of users who obtain their desired file by the cellular mode for the remaining $m-n$ files is

$$
N_{\mathrm{BS} 2}=\sum_{i=n+1}^{m} \rho\left|S^{\prime}\right| q_{i} \text {. }
$$

Further, the number of cellular links for all files can be formulated as

$$
N_{\mathrm{BS}}=\sum_{i=1}^{n} \rho\left|S^{\prime}\right|\left(1-p_{i}\right) q_{i} e^{-|S| \rho p_{i}}+\sum_{i=n+1}^{m} \rho\left|S^{\prime}\right| q_{i} .
$$

Therefore, EE of the converged network in this case can be given as follows:

$$
\begin{aligned}
\eta(n) & =\sum_{i=1}^{n} \pi a^{2} \rho\left(1-p_{i}\right) q_{i}\left(-\frac{1}{\ln 2}\right) \\
& \cdot\left\{\frac{\left(1-e^{-\pi r^{2} a^{2} \rho p_{i}}\right) e^{\sigma_{n}^{2} r^{\alpha} a^{\alpha} / 2 P_{d}} E_{i}\left(-\sigma_{n}^{2} r^{\alpha} a^{\alpha} / 2 P_{d}\right)}{\left(P_{d}+P_{u}\right)}\right. \\
& \left.+e^{-\pi r^{2} a^{2} \rho p_{i}} e^{\sigma_{n}^{2} a^{\alpha} / 2 P_{t}} E_{i}\left(-\frac{\sigma_{n}^{2} a^{\alpha} / 2 P_{t}}{\left(P_{t}+P_{c}\right)}\right)\right\} \\
& +\sum_{i=n+1}^{m} \rho S^{\prime} q_{i} e^{\sigma_{n}^{2} a^{\alpha} / 2 P_{t}} E_{i}\left(-\frac{\sigma_{n}^{2} a^{\alpha} / 2 P_{t}}{\left(P_{t}+P_{c}\right)}\right) .
\end{aligned}
$$

In order to obtain the maximum energy efficiency, we should find the optimal number of cache files $n$ in the virtual cache. The optimization problem is

$$
\begin{aligned}
\arg \max _{n} & \eta(n) \\
\text { s.t. } & 1 \leq n \leq m .
\end{aligned}
$$

However, it is drastically difficult to find the optimal $n_{\text {opt }}$ in an analytical way. Since $n$ is a discrete variable, we can substitute $n \in\{1,2, \ldots, m\}$ into the problem and perform an exhaustive searching to get the solution.

\section{Numerical Results}

In this section, numerical results are provided to investigate the effects of the system parameters. The radius of the cellular 


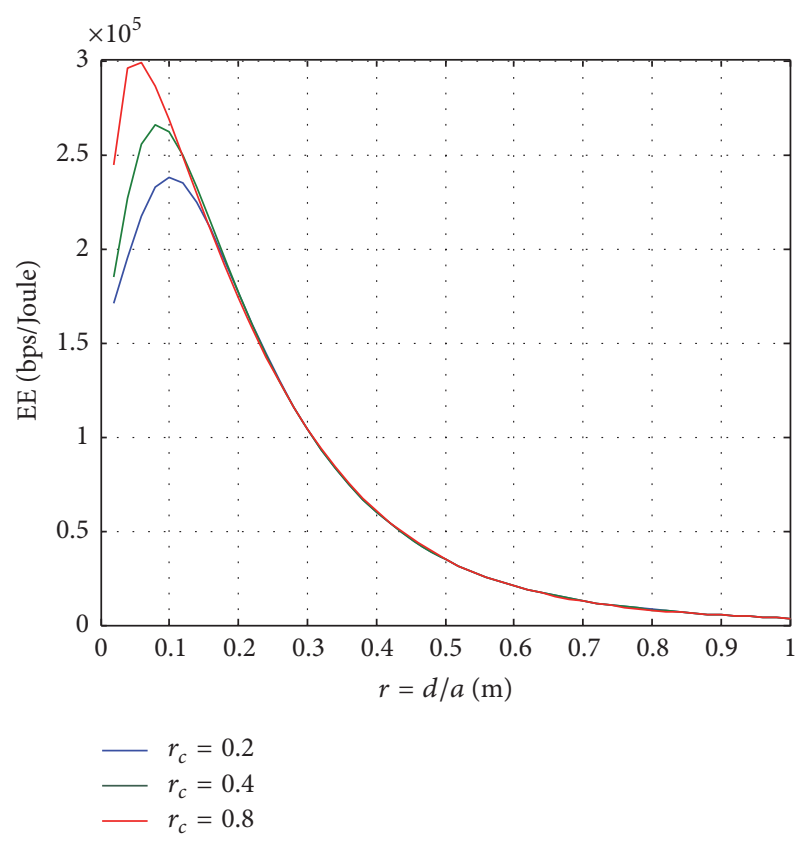

FIGURE 3: EE versus the collaboration distance $d$.

is $2000 \mathrm{~m}$; that is, $a=2000$, and the average intensity of PPP distribution of users in the cell is $\rho=0.003$ and the number of all files is $m=1000$. The circuit power is set to be $0.1 \mathrm{~W}[9,12]$. We set transmit power to be $20 \mathrm{dBm}$ for all D2D users and $46 \mathrm{dBm}$ for cellular transmitter. The power of additive white Gaussian noise is $-97.6 \mathrm{dBm}$. The Zipf distribution parameter of user requesting files is set as $r_{q}=1$ in all simulations.

First, we want to find the optimal collaboration distance which can maximize the EE of the converged network. We show the EE of the converged network versus the collaboration distance $d$ in Figure 3. Obviously, there exists a unique optimal collaboration distance $d$ for various $r_{c}$. Thus, if the system parameters of our converged network are available, we can solve the optimization problem to find the optimal collaboration distance. The larger $r_{c}$ is, the smaller $d_{\text {opt }}$ is. In other words, the optimal collaboration distance $d_{\text {opt }}$ decreases as $r_{c}$ increases. The reason is that, in a larger $r_{c}$ case, there are more popular files stored around the requesting user. Hence, the probability of finding it within the collaboration distance becomes larger. In addition, when $r>0.15$, we can see that these three systems have almost the same EE. The reason is that if the D2D communication range increases, the probability of finding a file from the $\mathrm{D} 2 \mathrm{D}$ users also increases. When the D2D communication distance is large enough, the probability of the event that D2D mode is only activated converges towards 1 . Therefore, the EEs of the converged systems with different $r_{c}$ have the same results. In Figure 4, we show the effect of the number of cache files in the virtual cache $n$ on the energy efficiency of converged network. Similarly, we can see that there exist a unique optimal number of cache files, namely, $n_{\mathrm{opt}}$ from our simulation. Thus, given the system parameters, we also can compute the optimal number of caching files. For a given $n$, the smaller $r_{c}$ incurs lower EE. It means the more intensive the popularity

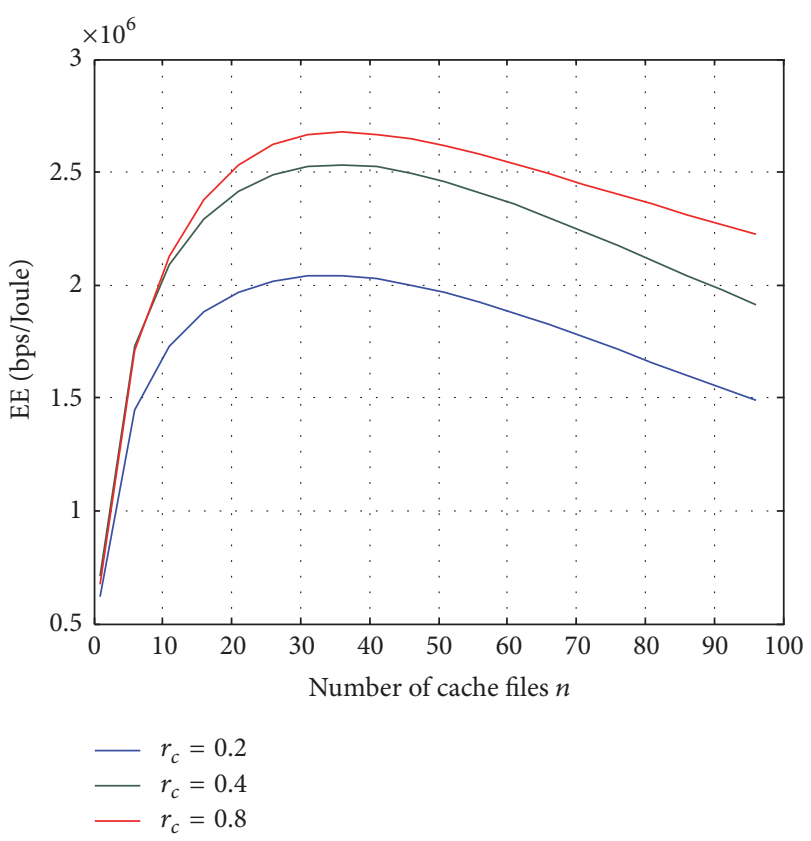

Figure 4: EE versus the number of cache files $n$.

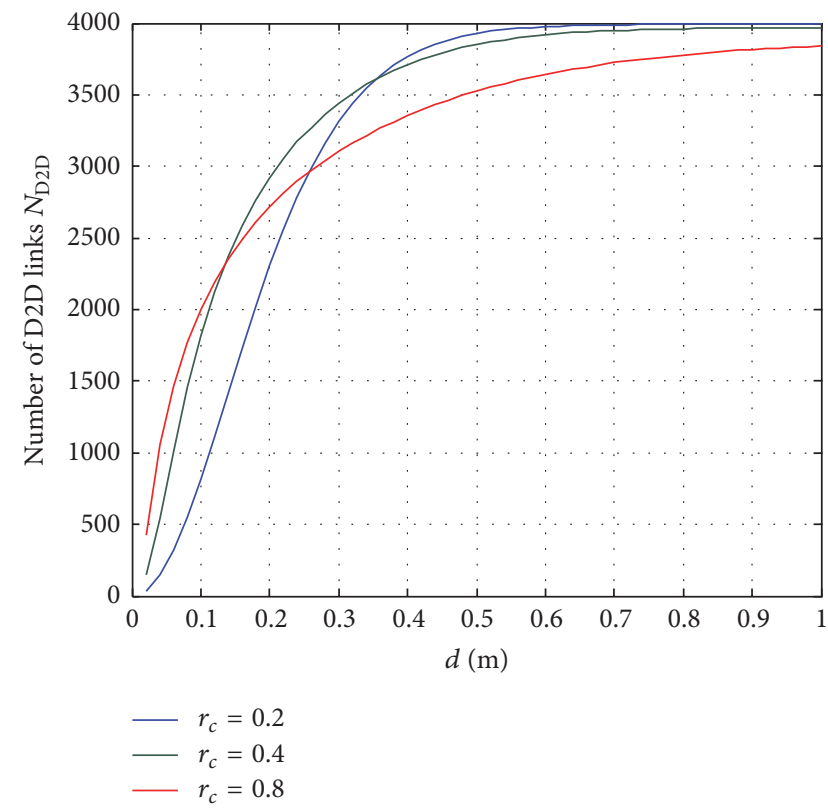

FIGURE 5: $N_{\text {D2D }}$ versus the collaboration distance $d$.

of video files is, the higher EE the converged network obtains.

Figure 5 shows the number of active D2D links $N_{\mathrm{D} 2 \mathrm{D}}$ versus the normalized collaboration distance $r$. We can observe that $N_{\mathrm{D} 2 \mathrm{D}}$ increases rapidly as $r$ increases. The larger $r$ means that the probability of finding the requested files becomes larger. So more D2D links are activated. As $r$ increases further, the number of active $\mathrm{D} 2 \mathrm{D}$ links $N_{\mathrm{D} 2 \mathrm{D}}$ remained stable. It is worth pointing out that users can find all of their requested files in the D2D virtual cache when the D2D collaboration distance is greater than a certain threshold. Furthermore, we 


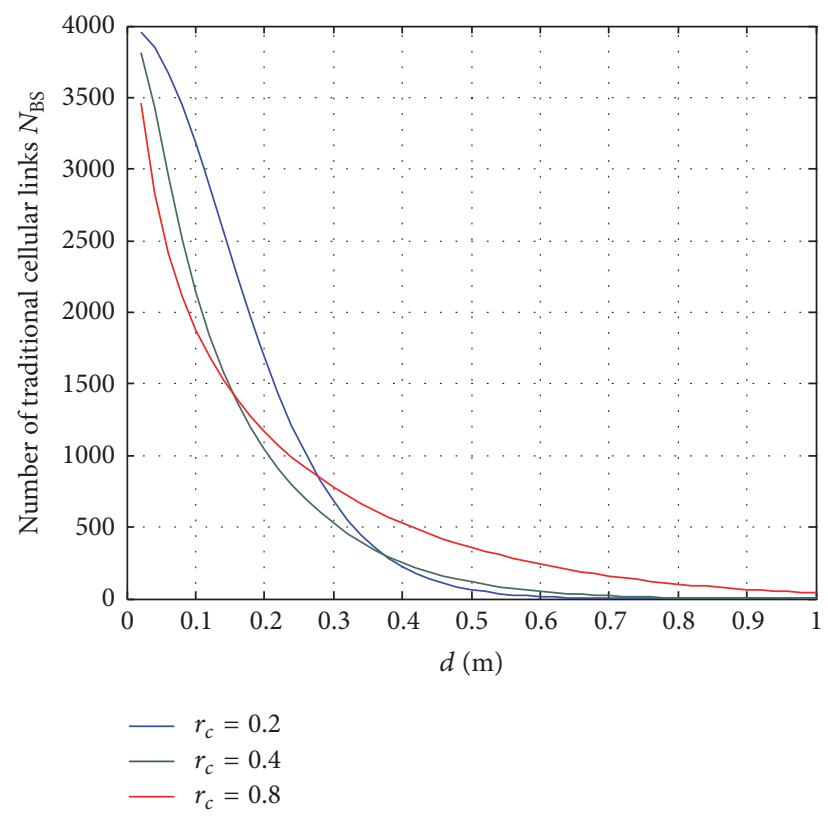

FIGURE 6: $N_{\mathrm{BS}}$ versus the collaboration distance $d$.

can see that the larger $r_{c}$ is, the lower the increasing rate of the number of D2D links is. Moreover, when $d<0.1$, the system with $r_{c}=0.8$ has the most D2D links compared to the systems with $r_{c}=0.4,0.2$. On the contrary, when $d>0.4$, the system with $r_{c}=0.2$ has the most D2D links compared to other systems. Figure 6 shows the number of traditional cellular links $N_{\mathrm{BS}}$ versus the normalized collaboration distance $r$. The trend of $N_{\mathrm{BS}}$ versus the collaboration distance $r$ is in contrast with that of $N_{\mathrm{D} 2 \mathrm{D}}$. The reason is that if D2D mode is not feasible, the user has to work in the cellular mode. Then, as the number of D2D links in the converged network increases, the number of cellular links decreases. That is why $N_{\mathrm{D} 2 \mathrm{D}}$ and $N_{\text {BS }}$ have a complementary relationship in Figures 5 and 6.

\section{Conclusion}

In this paper, we establish a new paradigm of wireless video delivery between users in the converged network of D2D communication and cellular network. In the proposed converged network, a user obtains its requested video file through either D2D link or traditional cellular link. The energy efficiency of the converged network is derived. What is more, we also study the effects of two key parameters: the collaborative distance $d$ and the number of cache files $n$ in the large virtual cache on energy efficiency. Finally, through simulations, we show that there indeed exist optimal collaborative distance and number of cache files to maximize the EE in the D2D-cellular converged network.

\section{Competing Interests}

The authors declare that they have no competing interests.

\section{Acknowledgments}

This work was in part supported by Natural Science Foundation of China (no. 61302093); the Open Research Fund of National Mobile Communications Research Laboratory, Southeast University (no. 2011D14, no. 2013D07); the Fundamental Research Funds for the Central Universities; Natural Science Basic Research Plan in Shaanxi Province of China, no. 2015JQ6234.

\section{References}

[1] N. Golrezaei, A. F. Molisch, A. G. Dimakis, and G. Caire, "Femtocaching and device-to-device collaboration: a new architecture for wireless video distribution," IEEE Communications Magazine, vol. 51, no. 4, pp. 142-149, 2013.

[2] N. Golrezaei, P. Mansourifard, A. F. Molisch, and A. G. Dimakis, "Base-station assisted device-to-device communications for high-throughput wireless video networks," IEEE Transactions on Wireless Communications, vol. 13, no. 7, pp. 3665-3676, 2014.

[3] L. Goratti, K. M. Gomez, R. Fedrizzi, and T. Rasheed, "A novel device-to-device communication protocol for public safety applications," in Proceedings of the IEEE Globecom Workshops, pp. 629-634, December 2013.

[4] M. Usman, A. A. Gebremariam, U. Raza, and F. Granelli, "A software-defined device-to-device communication architecture for Public Safety Applications in 5G Networks," IEEE Access, vol. 3, pp. 1649-1654, 2015.

[5] Y. Shen, C. Jiang, T. Q. S. Quek, and Y. Ren, "Device-to-device-assisted commuications in cellular network: an energy efficient approach in downlink video sharing scenario," IEEE Transactions on Wireless Communications, vol. 15, no. 2, pp. 1575-1587, 2015.

[6] X. Y. Qiu, L. Xuewen, K. Dong, and S. Zhu, "Energy efficiency analysis in device-to-device communication underlaying cellular networks," in Proceedings of the IEEE 10th Consumer Communications and Networking Conference (CCNC '13), pp. 625-630, IEEE, Las Vegas, Nev, USA, January 2013.

[7] H. H. Yang, J. Lee, and T. Q. S. Quek, "Heterogeneous cellular network with energy harvesting-based D2D communication," IEEE Transactions on Wireless Communications, vol. 15, no. 2, pp. 1406-1419, 2016.

[8] Y. Pei and Y.-C. Liang, "Resource allocation for device-to-device communications overlaying two-way cellular networks," IEEE Transactions on Wireless Communications, vol. 12, no. 7, pp. 3611-3621, 2013.

[9] C. Xu, L. Y. Song, Z. Han, D. Li, and B. Jiao, "Resource allocation using a reverse iterative combinatorial auction for deviceto-device underlay cellular networks," in Proceedings of the IEEE Global Communications Conference (GLOBECOM '12), pp. 4542-4547, Anaheim, Calif, USA, December 2012.

[10] H. Ahlehagh and S. Dey, "Hierarchical video caching in wireless cloud: approaches and algorithms," in Proceedings of the IEEE International Conference on Communications (ICC '12), pp. 7082-7087, Ottawa, Canada, June 2012.

[11] B. Peng, T. Peng, Z. Liu, Y. Yang, and C. Hu, "Cluster-based multicast transmission for device-to-device $\left(\mathrm{D}_{2} \mathrm{D}\right)$ communication," in Proceedings of the IEEE 78th Vehicular Technology Conference (VTC Fall '13), pp. 1-5, Las Vegas, Nev, USA, September 2013.

[12] N. Privault, Spatial Poisson Processes, Springer, Singapore, 2013. 
[13] M. Cha, H. Kwak, P. Rodriguez, Y.-Y. Ahnt, and S. Moon, "I tube, you tube, everybody tubes: analyzing the world's largest user generated content video system," in Proceedings of the 7th ACM SIGCOMM Internet Measurement Conference (IMC '07), pp. 1-14, October 2007.

[14] C. Yang, X. Xu, J. Han, and X. Tao, "Energy efficiencybased device-to-device uplink resource allocation with multiple resource reusing," Electronics Letters, vol. 51, no. 3, pp. 293-294, 2015.

[15] F. Baccelli, B. Blaszczyszyn, and P. Muhlethaler, "An Aloha protocol for multihop mobile wireless networks," IEEE Transactions on Information Theory, vol. 52, no. 2, pp. 421-436, 2006.

[16] H. J. Kang and C. G. Kang, "Mobile Device-to-Device (D2D) content delivery networking: a design and optimization framework," Journal of Communications and Networks, vol. 16, no. 5, pp. 568-577, 2014. 

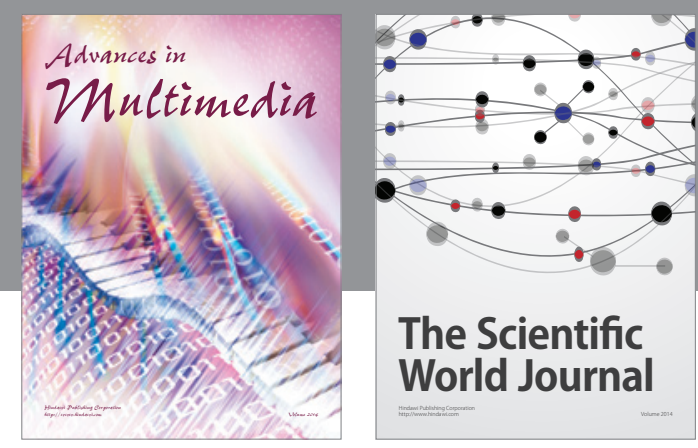

The Scientific World Journal
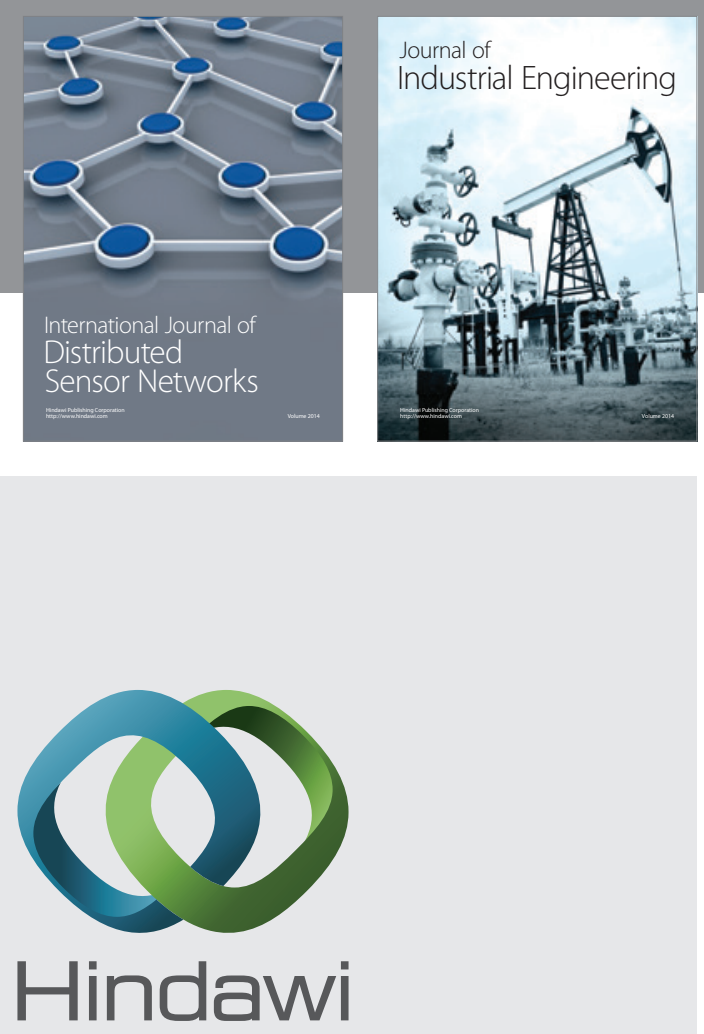

Submit your manuscripts at

http://www.hindawi.com

\section{Computer Networks} and Communications
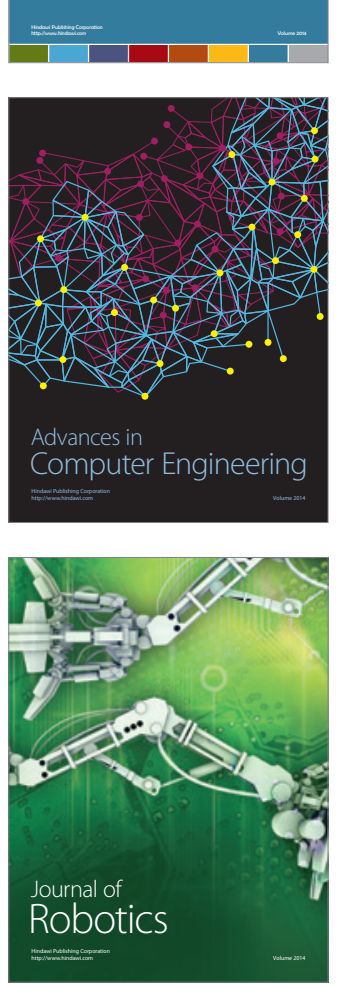
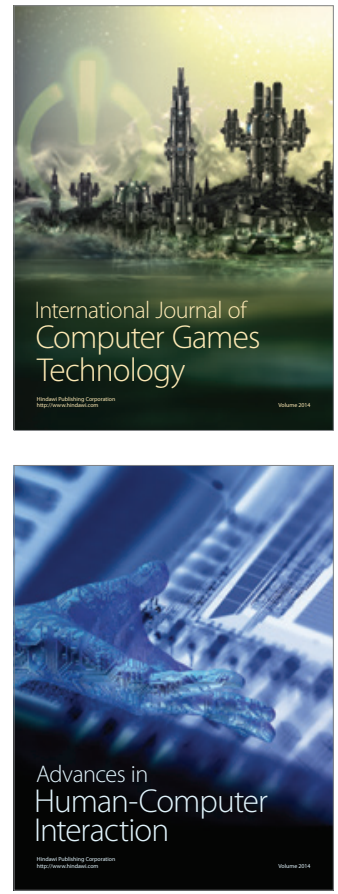
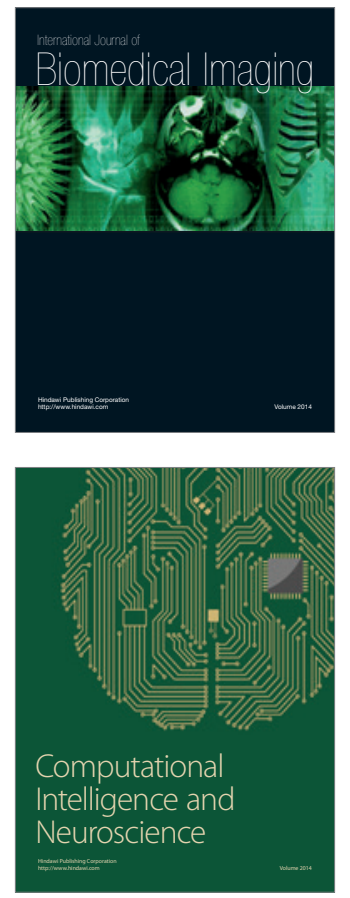
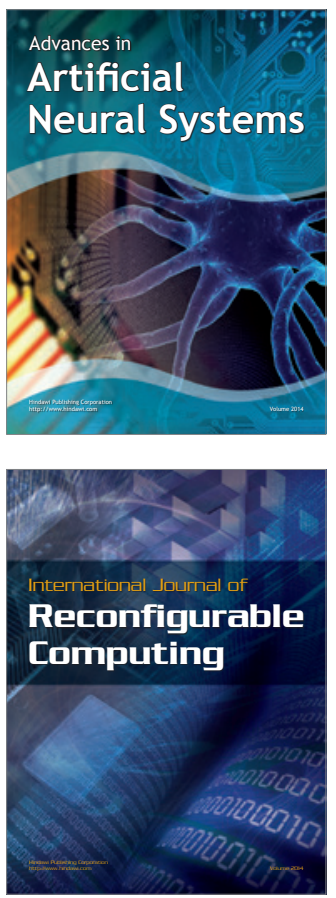
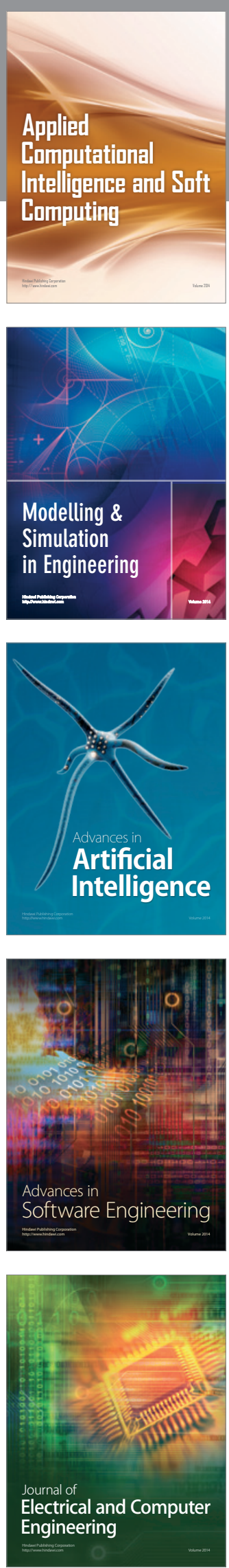